\title{
Copy-number variation analysis in familial nonsyndromic congenital anomalies of the kidney and urinary tract: Evidence for the causative role of a transposable element-associated genomic rearrangement
}

\author{
EKATERINI SIOMOU ${ }^{1}$, ARTEMIS G. MITSIONI ${ }^{2}$, VASILEIOS GIAPROS ${ }^{3}$, \\ IOANNA BOUBA $^{2}$, DIMITRIOS NOUTSOPOULOS ${ }^{4}$ and IOANNIS GEORGIOU ${ }^{2}$

\footnotetext{
${ }^{1}$ Department of Pediatrics, University Hospital of Ioannina; ${ }^{2}$ Laboratory of Medical Genetics and Human Reproduction, Faculty of Medicine, University of Ioannina; ${ }^{3}$ Neonatal Intensive Care Unit, University Hospital of Ioannina;

${ }^{4}$ Laboratory of General Biology, Faculty of Medicine, University of Ioannina, Ioannina 45500, Greece
}

Received October 17, 2016; Accepted March 23, 2017

DOI: $10.3892 / \mathrm{mmr} .2017 .6462$

\begin{abstract}
Most congenital anomalies of the kidney and urinary tract (CAKUT) are sporadic, but familial occurrence has been described, suggesting a genetic contribution. Copy-number variations (CNVs) were detected in patients with CAKUT to identify possible novel genomic regions associated with CAKUT. CNVs were investigated in 7 children with CAKUT from three unrelated families using array comparative genomic hybridization: female monozygotic twins with bilateral duplex collecting system/vesicoureteral reflux (VUR)/unilateral renal hypodyspasia (URHD); two male siblings with VUR/URHD; 3 male second cousins, one with bilateral VUR/URHD, one with bilateral VUR and one with ureterovesical junction obstruction (UVJO). Five patients had a normal constitution of CNVs, one had a duplication of $0.2 \mathrm{Mb}$ on the $5 \mathrm{q}$-arm (5q23.3), probably unrelated to CAKUT, and one with UVJO had a $1.4 \mathrm{Mb}$ deletion on the $17 \mathrm{q}$-arm (17q12) which includes a known CAKUT gene, $H N F 1 B$. The phenotype of $H N F 1 B$ deletion was extended including renal magnesium wasting. A higher coverage in transposable elements (TEs) was found in the deleted region compared with the expected density in any random genomic region. Notably, the $5^{\prime}$ breakpoint was mapped within a solo long terminal repeat (LTR) sequence. Moreover, highly similar members of solo LTR and mammalian interspersed
\end{abstract}

Correspondence to: Professor Ekaterini Siomou, Department of Pediatrics, University Hospital of Ioannina, Stavros Niarchos Avenue, Ioannina 45500, Greece

E-mail: eksiomou@yahoo.gr

Key words: copy number variations, familial congenital anomalies of the kidney and urinary tract, $H N F 1 B$ gene, transposable elements, comparative genomic hybridization, ureterovesical junction obstruction repetitive (MIR) elements, as well as nucleotide sequence microhomology were detected at the breakpoint regions. In conclusion, the deletion detected in one patient suggests this genomic imbalance as causative for UVJO. A not very well known phenotype of $H N F 1 B$ deletion resulting in both low urinary tract malformations and renal wasting of magnesium was described. The high load in TEs of the deleted region, the presence of highly similar elements, and the microhomology found at breakpoint regions may have contributed to the generation of the deletion. CNV analysis could reveal novel causative genomic regions in patients with CAKUT, and further studies in larger cohorts are needed.

\section{Introduction}

The prevalence of congenital anomalies of the kidney and urinary tract (CAKUT) is 17.7:1,000 live births (1). CAKUT constitute the major cause of chronic renal failure in childhood (2) and can occur in isolation (nonsyndromic CAKUT), or in association with other organ system malformations (2). While most cases are sporadic, a familial aggregation has been described in up to $15 \%$ of cases, pointing to a genetic contribution. In a recent study, 37 different heterozygous mutations (33 novel mutations) in 12 of 17 known CAKUT-causing genes were identified in $6.3 \%$ of 650 unrelated families with CAKUT. It thus appears that CAKUT is a genetically heterogeneous disease with diverse clinical phenotypes (3).

Submicroscopic chromosomal imbalances (deletions or duplications), known as copy-number variations (CNVs), have been used to identify novel genomic regions associated with CAKUT. CNVs were detected in $10.1 \%$ of 178 patients with CAKUT and were found to be inherited in $90 \%$ (9/10) of the families in which they were identified (4).

Transposable elements (TEs) can sculpt genome structure, with a profound contribution to genetic variation, through single nucleotide variations, CNVs (indels) or larger structural variations (5). Such TE-mediated rearrangements can be either active, as a direct result of retrotransposition events, or passive 
due to their repetitive nature $(6,7)$, causing diseases in some cases (7).

The aim of the present study was to investigate CNVs in a series of children with nonsyndromic upper and lower urinary tract anomalies, and to search for evidence of a possible causative role of a transposable element-associated genomic rearrangement. The novelty of the study is that it focused on first and second degree relatives with the same or similar CAKUT phenotype.

\section{Materials and methods}

Patients. Three unrelated families, each with at least two members diagnosed with nonsyndromic CAKUT in the Pediatric Nephrology Department of the University Hospital of Ioannina (Greece) were invited to participate in the study. The first family consisted of female monozygotic twins with CAKUT. One twin had a bilateral completely duplex collecting system, bilateral vesicoureteral reflux (VUR) grade III-IV in the lower pole, and right renal hypodysplasia (RHD) (relative function $38 \%$ ). The second twin had a left incompletely duplicated system (the two ureters joined just before entering the bladder), bilateral VUR grade II-III, and left RHD (relative function 38\%). The second family consisted of two male siblings one of which had bilateral VUR grade III and left RHD (relative function 26\%) and the second bilateral VUR grade III-IV. In the third family three males, second cousins, had CAKUT. One had bilateral VUR grade V (normal urethra) and right RHD (relative function 28\%), one had bilateral VUR grade III, and the third had severe ureterovesical junction obstruction (UVJO), requiring surgical intervention. The patients of the third family were members of a cohort of gypsies with a high rate of inbreeding (first cousin marriages).

All the patients had normal laboratory findings, apart from the 15-year-old patient with UVJO from the gypsy family who had persistent hypomagnesemia (serum magnesium levels: 1.02-1.17 mEq/1, normal range 1.3-2.1 mEq/l), hypermagnesuria [fractional magnesium excretion (FeMg): 5.0-5.5\%, and hypocalciuria (fractional calcium excretion: $0.18-0.2 \%$ ) [in patients with hypomagnesemia, hypermagnesuria has been defined as FeMg $>4$ (8), and hypocalciuria as fractional calcium excretion $<1 \%$ ) (9)]. Serum creatinine, calcium, phosphate, albumin, parathyroid hormone, 25-hydroxyvitamin D levels, and electrolytes were within normal limits.

The CAKUT in these children were investigated and characterized by urinary tract ultrasound, voiding cystourethrography, dimercaptosuccinic acid (DMSA) scan, and technetium-99 m mercaptoacetyltriglycine (MAG3) scan, as indicated. VUR was classified into grades I-V according to the International Reflux Classification (10). RHD was diagnosed in kidneys with reduced renal length and regular outline, with or without cortical hyperechogenicity and loss of corticomedullary differentiation on ultrasound, and with persistent (for $\geq 6$ months), general reduction in 99mTc-DMSA uptake (relative function $<45 \%$ ).

$C N V$ detection. For the genetic study, peripheral venous blood was collected for genomic DNA extraction, according to the manufacturer's protocol using QIAamp DNA Mini Kit (Qiagen, Hilden, Germany), at the time of routine blood testing of the patients. DNA samples were investigated for the presence of CNVs, using array-comparative genomic hybridization (CGH). Array-CGH was performed by Cytochip ISCA array (Blue Gnome-version 1.0) with 180,000 oligos, analyzed using buildGRCh37 (hg19).

The following resources were used for the analysis of the cases: Ensemble (http://www.ensembl.org), Database of Genomic Variants (http://projects.tcag.ca/variation/), UCSC Genome Bioinformatics Site (http://genome.ucsc.edu/), and Online Mendelian Inheritance In Man (http://ncbi.nlm.nih .gov/omim).

CNVs $>0.2 \mathrm{Mb}$ were considered significant. CNVs that did not contain genes, or were $<0.2 \mathrm{Mb}$ (unless containing a gene of known pathogenic significance) were considered of normal constitution.

Bioinformatics. TE sequences coverage was measured using the in silico program RepeatMasker (http://www .repeatmasker.org/), and UCSC genome browser to extract nucleotide sequences from the human genome (GRCh38). Sequence features were analyzed and visualized in the UCSC browser. Nucleotide sequence alignments were performed using the Blast algorithm (http://blast.ncbi.nlm.nih.gov/Blast.cgi). Breakpoint regions (200 bp stretches surrounding the breakpoints) were analyzed using the Blast algorithm for the identification of sequence homology.

\section{Results}

Of the 7 study children with familial nonsyndromic CAKUT, 5 had a normal constitution of CNVs (well-established polymorphism variants).

One sibling from the second family, with bilateral VUR grade III and left RHD had a $0.2 \mathrm{Mb}$ duplication on the long arm of chromosome 5 (5q-arm) at chromosomal band 5q23.3. The duplication partially disrupts the Chondroitin Sulfate Sulthase 3 (CHY3) gene (Fig. 1A). His brother, with a similar CAKUT (bilateral VUR grade III-IV), did not harbor the same CNV.

One male teenager, from the gypsy family, with UVJO, was found to have a deletion approximately $1.4 \mathrm{Mb}$ in size on the long arm of chromosome 17 (17q-arm) at chromosomal band 17q12. This deletion consists of two OMIM disease genes, the Acetyl-CoaCarboxylase-Alpha (ACACA, OMIM\#200350), and the Hepatocyte Nuclear Factor-1-Beta (HNF1B, OMIM\#189907) (Fig. 1B).

To gain insight into the possible molecular mechanism underlying the deletion detected, we measured the load in TE sequences within the deleted region. A higher coverage $(51.33 \%)$ was found than expected when inspecting any random genome region (Fig. 2A). Notably, the $5^{\prime}$ breakpoint was mapped in a solo long terminal repeat (LTR) sequence (MLT1J2), sharing high similarity (76\%) to the solo LTR sequence MamRep1527 located at the 3 ' breakpoint region. Moreover, a significant load in highly similar (70-89\%) mammalian interspersed repetitive (MIR) element sequences, namely MIRb and MIRc, was found in the $20 \mathrm{~Kb}$-region bilateral to the 5 ' and 3 ' breakpoints, amounting to 11.87 and $5.25 \%$, respectively (Fig. 2B). Finally, a $47 \%$ nucleotide sequence homology was identified in a stretch of $200 \mathrm{bp}$ surrounding the breakpoints, while 
A
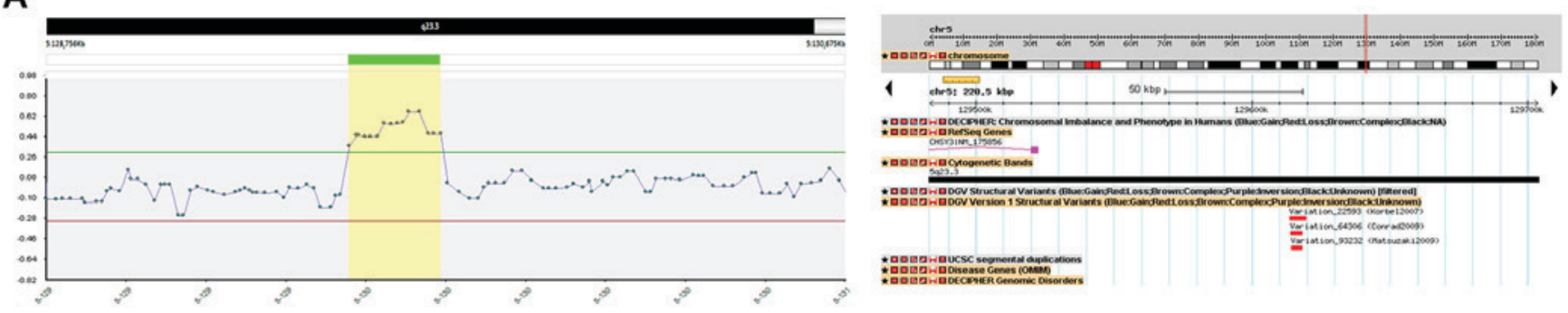

B
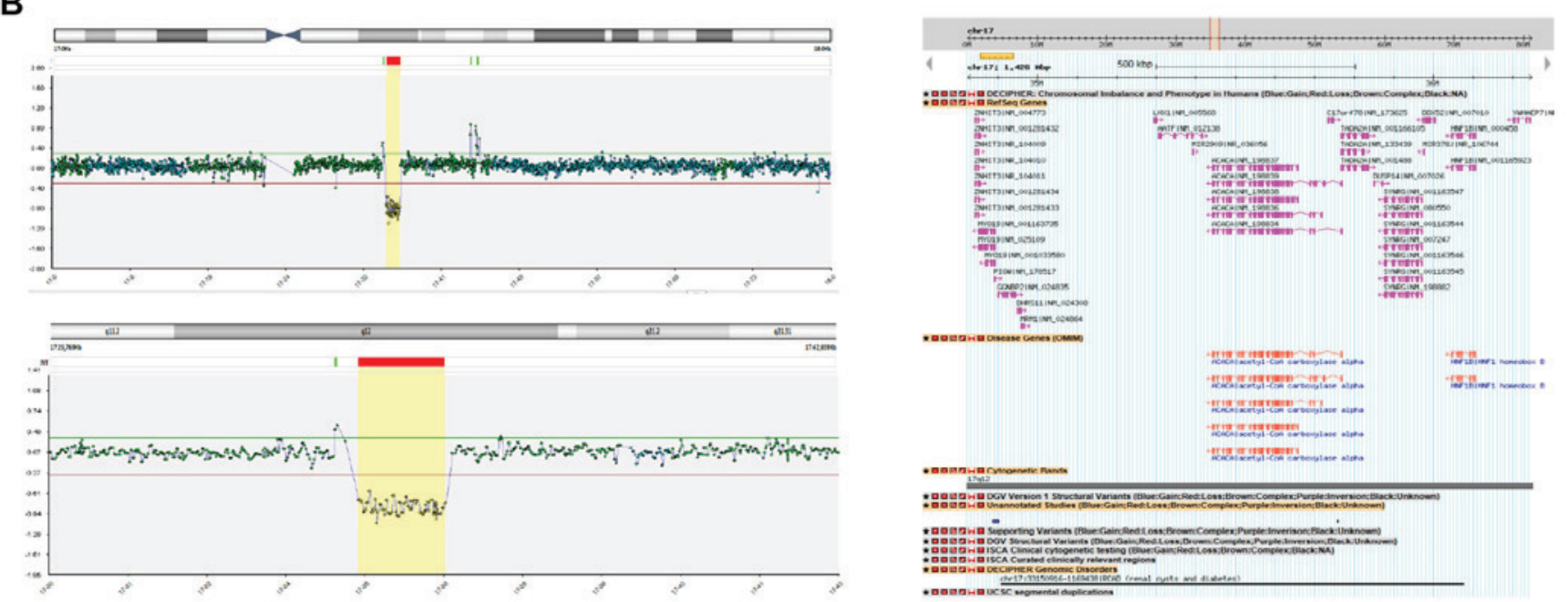

Figure 1. Genomic rearrangements in patients with CAKUT. (A) Duplication of $0.2 \mathrm{Mb}$ in size on the long arm of chromosome 5 (5q-arm) at chromosomal band 5q23.3; (B) Deletion of approximately 1.4 Mb in size on the long arm of chromosome 17 (q-arm) at chromosomal band 17q12 (location: 34,823,154-36,248,888).

no microhomology was found in the breakpoint junctions (Fig. 2C and D).

CNVs were not studied in the parents of the affected children, but all the parents were investigated by urinary tract US and had no CAKUT phenotype.

\section{Discussion}

This study investigated the presence of CNVs in a series of 7 children with familial nonsyndromic CAKUT. Its novelty is the focus on first and second degree relatives with the same or similar nonsyndromic CAKUT phenotype, and on evidence for the causative role of a transposable element-associated genomic rearrangement.

CNV associated with CAKUT was detected in one of the 7 patients (14\%). The number of patients investigated was very small, but the rate of CNVs was in agreement with previous studies in which CNVs were identified in $16.6 \%$ (11) and $10.1 \%$ (4) in large numbers of patients with nonsyndromic CAKUT (522 and 178 respectively). Sanna-Cherchi and colleagues (11) studied patients with congenital solitary kidney and renal hypoplasia, while Caruana and colleagues (4) included a wide range of CAKUT, finding a high incidence of CNVs in cases of multicystic dysplastic kidney (MCDK) (30\%), and posterior urethral valves (24\%). Similarly, Westland and colleagues (12), using CNV analysis in 80 patients with a solitary functioning kidney, found genomic imbalances in 11 of $80(14 \%)$. Weber and colleagues (2) reported a similar CNV frequency $(10 \%)$ in 30 children with syndromic CAKUT.
The $0.2 \mathrm{Mb}$ duplication found in one sibling of the second family is not considered causative for his CAKUT phenotype, since it does not include any genes known to be associated with CAKUT (Fig. 1A), and his brother with the same CAKUT phenotype had a normal constitution of CNVs.

One child in the gypsy family, with UVJO, had a deletion approximately $1.4 \mathrm{Mb}$ in size on the long arm of chromosome 17q12. The deletion consists of two OMIM disease genes, the Acetyl-CoaCarboxylase-Alpha (ACACA, OMIM\#200350), and the Hepatocyte Nuclear Factor-1beta (HNF1B, OMIM\#189907) (Fig. 1B). The same CNV including the $H N F 1 B$ gene has also been reported recently by Caruana and colleagues (4) in a female infant with MCDK. The HNF1B gene has been associated with renal cyst, diabetes mellitus and genital malformation (RCAD syndrome: OMIM 137920), where a 75 bp deletion in exon 2 causes a loss-of-function mutation (13). HNF1B mutations are well described in patients with upper urinary tract malformations. Nakayama and colleagues (14) identified HNF1B mutations in 5 of 50 patients (10\%) with CAKUT (3 with MCDK and 2 with RHD), where de novo heterozygous complete deletions of $H N F 1 B$ were found in patients with MCDK. CNV analysis showed 1.4 Mb deletion of chromosome 7, involving the whole $H N F 1 B$ gene with breakpoints in flanking segmental duplications. Thomas and colleagues (15) reported that among 50 North American Caucasian children with RHD, 4 (8\%) carried mutations in $H N F 1 B$ gene. In our study, a CNV involving the whole $H N F 1 B$ gene was found in one patient with a lower urinary tract malformation (UVJO). Considering that the HNF1B is expressed in the ureteric bud 
A

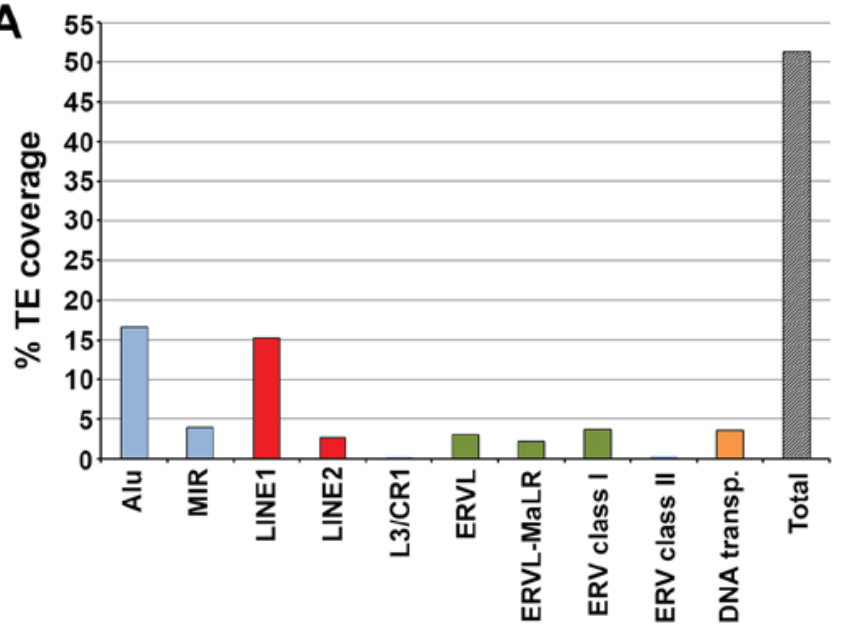

\begin{tabular}{|c|c|c|}
\hline \multicolumn{2}{|c|}{ Transposable element } & $\begin{array}{l}\text { Sequence } \\
\text { percentage }\end{array}$ \\
\hline \multirow{2}{*}{ SINES } & Alu & 16.52 \\
\hline & MIR & 3.92 \\
\hline \multirow[b]{2}{*}{ LINEs } & LINE1 & 15.30 \\
\hline & $\begin{array}{l}\text { LINE2 } \\
\text { L3/CR1 }\end{array}$ & $\begin{array}{l}2.64 \\
0.19\end{array}$ \\
\hline \multirow{4}{*}{ LTR elements } & ERVL & 2.97 \\
\hline & ERVL-MaLR & 2.19 \\
\hline & ERV class I & 3.74 \\
\hline & ERV class II & 0.29 \\
\hline \multirow{2}{*}{$\begin{array}{c}\text { DNA } \\
\text { elements }\end{array}$} & $\begin{array}{c}\text { DNA } \\
\text { transposons }\end{array}$ & 3.57 \\
\hline & & 51.33 \\
\hline
\end{tabular}

Breakpoint (5' end)

B
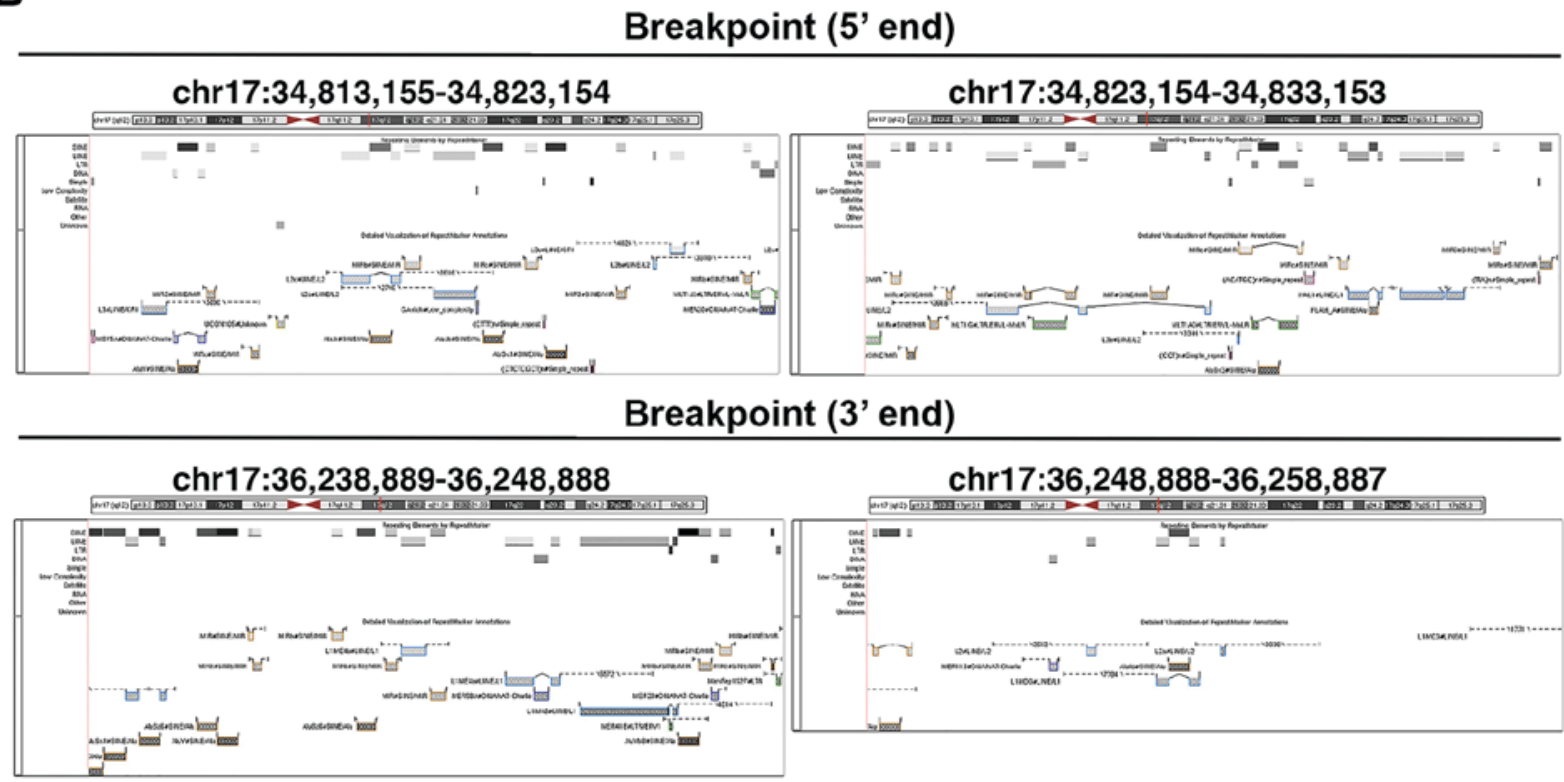

C

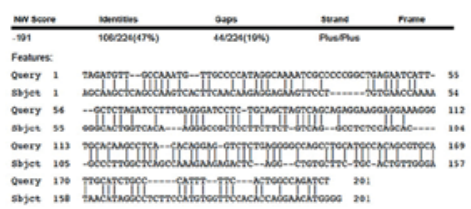

D

\section{Proximal $\overrightarrow{\text { сстстGCAGCTAGTCAGCAGAGGAAGGAGGAAAGGGTGCACAAGCCTCAC }}$ \\ 17q del сCTCTGCAGCTAGTCAGCAGAGGAACACGCCCTTGGCTCAGCCAAAGAAG \\ Distal \\ TCCTTCTTCTGTCAGGCCTCTCCAGCACGCCCTTGGCTCAGCCAAAGAAG}

Figure 2. Bioinformatic analysis of the 17q12-deleted region in a patient with CAKUT. (A) Abundance in transposable element sequences within the 17q12-deleted region; (B) Abundance in transposable element sequences within the $20 \mathrm{~Kb}$-region bilateral to the 5' and 3' breakpoints; (C) Alignment of nucleotide sequence of $200 \mathrm{bp}$ surrounding the proximal and distal breakpoints; (D) Alignment of the normal proximal and distal DNA sequences as well as the pathogenic variant $(17 \mathrm{q} 12 \mathrm{del})$. Nucleotide sequence homology to the normal proximal and distal wild-type sequences is shown in blue and red. Arrow extending to the right indicates that MLT1J2 solo LTR is longer than the sequence shown.

derivatives $(16,17)$, this finding is not surprising. The association of HNFIB alteration with the UVJO phenotype has not been reported as often as its association with upper urinary tract malformations. Adalat et al (18) reported a mutation in the $H N F 1 B$ gene in one patient with hydronephrosis/hydroureter. Specifically, they found heterozygous mutations in $H N F 1 B$ gene in $23 \%$ of 91 cases with renal malformation. The range of phenotypes included large bright kidneys, MCDK, and hydronephrosis/hydroureter. One patient has been reported with complete prune-belly syndrome associated with a complete $H N F 1 B$ gene deletion (19).
Our patient had also persistent hypomagnesemia, hypermagnesuria and hypocalciuria. Adalat et al (18) found that $44 \%$ of the $18 H N F 1 B$ mutation carriers had hypomagnesemia. They detected highly conserved HNF1-recognition sites in FXYD2, and demonstrated HNF1B-mediated transactivation of FXYD2, a gene that can cause autosomal dominant hypomagnesemia, hypermagnesuria and hypocalciuria when mutated (20). Our findings, even though not novel, depict a phenotype of $H N F 1 B$ gene alternation, which includes both lower urinary tract malformations and renal magnesium wasting. Therefore, our patient's phenotypic features both overlap and expand on 
reported cases of nonsyndromic CAKUT. Given, however, that the two other family members (cousins) with CAKUT did not have the 17q12 deletion, we suggest that either the CAKUT in this family is unrelated or that the UVJO and the VUR in the cousins are due to a separate undetected genetic defect. Considering that these patients had a highly consanguineous background, a possibly recessive cause of CAKUT cannot be excluded. It should be pointed out that the etiology of the majority of CAKUT cases remains unknown. The genomic imbalance, such as copy number variants, genomic, or de novo mutations, can explain up to one-third of all CAKUT cases. Moreover, findings from several studies suggest a contribution of epigenetic and environmental factors on the pathogenesis of CAKUT, supporting the theory of its multifactorial character (21).

Congenital diseases, such as CAKUT, have developmental origin (22), and TEs and their control mechanisms regulate development (23). It is known that TEs are involved in recombination events, representing a major source of structural variation in the genomic landscape (24). Even if deletions of chromosome $17 \mathrm{q} 12$ spanning $H N F 1 B$ gene are one of the most commonly identified mutations associated with CAKUT (21), their molecular characterization, and, especially, the coverage in TEs sequences within or at the boundaries of such deletions has not been previously reported. The novelty of our study was the bioinformatic analysis of a CAKUT-causative $1.4 \mathrm{Mb}$ deletion on the $17 \mathrm{q}$-arm based on the in silico program RepeatMasker, which effectively identifies TEs sequences within any given genomic region. An additional advantage of such approach comes from the use of the alignment heuristic program cross_match for the performance of sequence comparisons, providing a high sensitivity. Our analysis revealed: (a) a high coverage in TEs within the 17q12-deleted region (Fig. 2A), (b) a MLT1J2 solo LTR sequence at the 5 ' breakpoint (Fig. 2B), (c) the presence of 4.6- and 2-fold higher load of MIR sequences on either side of the 5' and 3' breakpoint regions respectively, compared to their expected genomic percentages (25) (Fig. 2B), and (d) $47 \%$ nucleotide sequence microhomology in a $200 \mathrm{bp}$ region surrounding the breakpoints (Fig. 2C). Based on these findings, it is tempting to suggest that TEs may have served as a substrate for the generation of the deletion. We believe that the deletion detected may have originated from the mispairing of TE sequences, possibly via non-allelic homologous recombination. Given that TEs can form non B-DNA structures promoting the formation of DNA nicks, double strand breaks or stall replication forks (26), we cannot exclude the involvement of microhomology-mediated replication-dependent recombination mechanisms, as previously reported in human $\mathrm{Xq}$ isochromosome formation (27).

In conclusion, among 7 children with familial nonsyndromic CAKUT, one with UVJO was found to have a significant CNV, suggesting this genomic imbalance as causative of the anomaly, since it includes a known CAKUT gene, $H N F 1 B$. The phenotype of the $H N F 1 B$ deletion was extended, and included both lower urinary tract malformation and renal magnesium wasting. Moreover, we determined the topological features, in terms of nucleotide sequence identity, of the deleted genomic region. Overall, our findings provide evidence of a correlation between a TE-associated genomic rearrangement and CAKUT. Future studies will shed more light for the role of TEs as causative factors of pathogenic variants. Finally, CNV analysis could reveal novel causative genomic regions in patients with CAKUT as well as in other multifactorial diseases, and further studies in larger cohorts are needed.

\section{References}

1. Melo BF, Aguiar MB, Bouzada MC, Aguiar RL, Pereira AK, Paixão GM, Linhares MC, Valerio FC, Simões E Silva AC and Oliveira EA: Early risk factors for neonatal mortality in CAKUT: Analysis of 524 affected newborns. Pediatr Nephrol 27: 965-972, 2012 .

2. Weber S, Landwehr C, Renkert M, Hoischen A, Wühl E, Denecke J, Radlwimmer B, Haffner D, Schaefer F and Weber RG: Mapping candidate regions and genes for congenital anomalies of the kidneys and urinary tract (CAKUT) by array-based comparative genomic hybridization. Nephrol Dial Transplant 26: 136-143, 2011 .

3. Hwang DY, Dworschak GC, Kohl S, Saisawat P, Vivante A, Hilger AC, Reutter HM, Soliman NA, Bogdanovic R, Kehinde EO, et al: Mutations in 12 known dominant disease-causing genes clarify many congenital anomalies of the kidney and urinary tract. Kidney Int 85: 1429-1433, 2014.

4. Caruana G, Wong MN, Walker A, Heloury Y, Webb N, Johnstone L, James PA, Burgess T and Bertram JF: Copy-number variation associated with congenital anomalies of the kidney and urinary tract. Pediatr Nephrol 30: 487-495, 2015.

5. Hancks DC and Kazazian HH Jr: Active human retrotransposons: Variation and disease. Curr Opin Genet Dev 22: 191-203, 2012.

6. Kidd JM, Graves T, Newman TL, Fulton R, Hayden HS, Malig M, Kallicki J, Kaul R, Wilson RK and Eichler EE: A human genome structural variation sequencing resource reveals insights into mutational mechanisms. Cell 143: 837-847, 2010.

7. Hancks DC and Kazazian HH Jr: Roles for retrotransposon insertions in human disease. Mob DNA 7: 9, 2016.

8. Elisaf M, Panteli K, Theodorou J and Siamopoulos KC: Fractional excretion of magnesium in normal subjects and in patients with hypomagnesemia. Magnes Res 10: 315-320, 1997.

9. Bianchetti MG, Edefonti A and Bettinelli A: The biochemical diagnosis of Gitelman disease and the definition of 'hypocalciuria'. Pediatr Nephrol 18: 409-411, 2003.

10. Medical versus surgical treatment of primary vesicoureteral reflux: Report of the International Reflux Study Committee. Pediatrics 67: 392-400, 1981.

11. Sanna-Cherchi S, Kiryluk K, Burgess KE, Bodria M, Sampson MG, Hadley D, Nees SN, Verbitsky M, Perry BJ, Sterken R, et al: Copy-number disorders are a common cause of congenital kidney malformations. Am J Hum Genet 91: 987-997, 2012.

12. Westland R, Verbitsky M, Vukojevic K, Perry BJ, Fasel DA, Zwijnenburg PJ, Bökenkamp A, Gille JJ, Saraga-Babic M, Ghiggeri GM, et al: Copy number variation analysis identifies novel CAKUT candidate genes in children with a solitary functioning kidney. Kidney Int 88: 1402-1410, 2015.

13. Lindner TH, Njolstad PR, Horikawa Y, Bostad L, Bell GI and Sovik O: A novel syndrome of diabetes mellitus, renal dysfunction and genital malformation associated with a partial deletion of the pseudo-POU domain of hepatocyte nuclear factor-1beta. Hum Mol Genet 8: 2001-2008, 1999.

14. Nakayama M, Nozu K, Goto Y, Kamei K, Ito S, Sato H, Emi M, Nakanishi K, Tsuchiya S and Iijima K: HNF1B alterations associated with congenital anomalies of the kidney and urinary tract. Pediatr Nephrol 25: 1073-1079, 2010.

15. Thomas R, Sanna-Cherchi S, Warady BA, Furth SL, Kaskel FJ and Gharavi AG: HNF1B and PAX2 mutations are a common cause of renal hypodysplasia in the CKiD cohort. Pediatr Nephrol 26: 897-903, 2011.

16. Coffinier C, Barra J, Babinet $C$ and Yaniv M: Expression of the vHNF1/HNF1beta homeoprotein gene during mouse organogenesis. Mech Dev 89: 211-213, 1999.

17. Kolatsi-Joannou M, Bingham C, Ellard S, Bulman MP, Allen LI, Hattersley AT and Woolf AS: Hepatocyte nuclear factor-1beta: A new kindred with renal cysts and diabetes and gene expression in normal human development. J Am Soc Nephrol 12: 2175-2180, 2001. 
18. Adalat S, Woolf AS, Johnstone KA, Wirsing A, Harries LW, Long DA, Hennekam RC, Ledermann SE, Rees L, van't Hoff W, et al: HNF1B mutations associate with hypomagnesemia and renal magnesium wasting. J Am Soc Nephrol 20: 1123-1131, 2009.

19. Murray PJ, Thomas K, Mulgrew CJ, Ellard S, Edghill EL and Bingham C: Whole gene deletion of the hepatocyte nuclear factor-1beta gene in a patient with the prune-belly syndrome. Nephrol Dial Transplant 23: 2412-2415, 2008.

20. Meij IC, Koenderink JB, van Bokhoven H, Assink KF, Groenestege WT, de Pont JJ, Bindels RJ, Monnens LA, van den Heuvel LP and Knoers NV: Dominant isolated renal magnesium loss is caused by misrouting of the $\mathrm{Na}(+), \mathrm{K}(+)$-ATPase gamma-subunit. Nat Genet 26: 265-266, 2000.

21. Nicolaou N, Renkema KY, Bongers EM, Giles RH and Knoers NV: Genetic, environmental, and epigenetic factors involved in CAKUT. Nat Rev Nephrol 11: 720-731, 2015.

22. Schedl A: Renal abnormalities and their developmental origin. Nat Rev Genet 8: 791-802, 2007.
23. Friedli M and Trono D: The developmental control of transposable elements and the evolution of higher species. Annu Rev Cell Dev Biol 31: 429-451, 2015

24. Babatz TD and Burns KH: Functional impact of the human mobilome. Curr Opin Genet Dev 23: 264-270, 2013.

25. Mandal PK and Kazazian HH Jr: SnapShot: Vertebrate transposons. Cell 135: 192.e1, 2008.

26. Pearson CE, Nichol Edamura K and Cleary JD: Repeat instability: Mechanisms of dynamic mutations. Nat Rev Genet 6: 729-742, 2005

27. Koumbaris G, Hatzisevastou-Loukidou H, Alexandrou A, Ioannides M, Christodoulou C, Fitzgerald T, Rajan D, Clayton S, Kitsiou-Tzeli S, Vermeesch JR, et al: FoSTeS, MMBIR and NAHR at the human proximal Xp region and the mechanisms of human Xq isochromosome formation. Hum Mol Genet 20: 1925-1936, 2011 